$\mathrm{J}$ o u r n a l of

Mathematics

and Applications

JMA No 39, pp 59-67 (2016)

\title{
About a Class of Analytic Functions Defined by Noor-Sălăgean Integral Operator
}

\section{Olga Engel and Yao Liang Chung}

ABstract: In this paper we intoduce a new integral operator as the convolution of the Noor and Sălăgean integral operators. With this integral operator we define the class $C_{N S}(\alpha)$, where $\alpha \in[0,1)$ and we study some properties of this class.

AMS Subject Classification: 30C45.

Keywords and Phrases: Noor integral operator; Sălăgean integral operator; Convolution.

\section{Introduction}

Let $U=\{z \in \mathbb{C}:|z|<1\}$ be the unit disk in the complex plane $\mathbb{C}$ and $H(U)$ denote the set of holomorphic (analytic) functions in $U$. We denote by

$$
\mathcal{A}=\left\{f \in H(U): f(0)=f^{\prime}(0)-1=0\right\}
$$

and

$$
S=\{f \in \mathcal{A}: f \text { is univalent in } U\} .
$$

We say that $f$ is starlike in $U$ if $f: U \rightarrow \mathbb{C}$ is univalent and $f(U)$ is a starlike domain in $\mathbb{C}$ with respect to origin. It is well-known that $f \in \mathcal{A}$ is starlike in $U$ if and only if

$$
\operatorname{Re}\left(\frac{z f^{\prime}(z)}{f(z)}\right)>0, \text { for all } z \in U \text {. }
$$

The class of starlike functions with respect to origin is denoted by $S^{*}$.

Let $T$ denote a subclass of $\mathcal{A}$ consisting of functions $f$ of the form

$$
f(z)=z-\sum_{j=2}^{\infty} a_{j} z^{j}
$$

COPYRIGHT (C) by Publishing House of Rzeszów University of Technology P.O. Box 85, 35-959 Rzeszów, Poland 
where $a_{j} \geq 0, j=2,3, \ldots$ and $z \in U$. A function $f \in T$ is called a function with negative coefficients. For the class $T$, the followings are equivalent [6]:
(i) $\sum_{j=2}^{\infty} j a_{j} \leq 1$,
(ii) $f \in T \cap S$,
(iii) $f \in T^{*}$, where $T^{*}=T \cap S^{*}$.

Let

$$
f(z)=z-\sum_{j=2}^{\infty} a_{j} z^{j}, a_{j} \geq 0, j=2,3, \ldots
$$

and

$$
g(z)=z-\sum_{j=2}^{\infty} b_{j} z^{j}, b_{j} \geq 0, j=2,3, \ldots
$$

then the convolution or the Hadamard product is defined by

$$
(f * g)(z)=z-\sum_{j=2}^{\infty} a_{j} b_{j} z^{j}=(g * f)(z), z \in U .
$$

The study of operators plays an important role in geometric function theory. For $f \in H(U), f(0)=0$ and $n \in \mathbb{N}_{0}=\mathbb{N} \cup\{0\}$, the $I_{S}^{n}$ Sălăgean integral operator is defined as follows [7]:

(i) $I_{S}^{0} f(z)=f(z)$,

(ii) $I_{S}^{1} f(z)=I f(z)=\int_{0}^{z} f(t) t^{-1} d t$,

(iii) $I_{S}^{n} f(z)=I_{S}\left(I_{S}^{n-1} f(z)\right)$.

We remark that if $f$ has the form (1.1), then

$$
I_{S}^{n} f(z)=z-\sum_{j=2}^{\infty} \frac{a_{j}}{j^{n}} z^{j}
$$

where $n \in \mathbb{N}_{0}$.

In [5] Noor defined an integral operator $I_{N}^{n}: \mathcal{A} \rightarrow \mathcal{A}$ as follows

$$
I_{N}^{n} f(z)=\frac{n+1}{z^{n}} \int_{0}^{z} t^{n-1} I_{N}^{n}(f(t)) d t
$$

where $n \in \mathbb{N}_{0}$.

Let $f_{n}(z)=\frac{z}{(1-z)^{n+1}}$ and let $f_{n}^{(-1)}(z)$ be defined such that 
We note that

$$
f_{n}^{(-1)}(z) * f_{n}(z)=\frac{z}{1-z}
$$

$$
I_{N}^{n} f(z)=f_{n}^{(-1)}(z) * f(z)=\left[\frac{z}{(1-z)^{n+1}}\right]^{(-1)} * f(z) .
$$

We remark that if $f$ has the form (1.1), then

$$
I_{N}^{n} f(z)=z-\sum_{j=2}^{\infty} \frac{a_{j}}{C(n, j)} z^{j}
$$

where $C(n, j)=\frac{(n+j-1) !}{n !(j-1) !}$.

\section{Preliminaries}

The following definitions and lemmas will be required in the sequel.

Definition 2.1. [2,3] Let $f$ and $g$ be analytic functions in $U$. We say that the function $f$ is subordinate to the function $g$, if there exist a function $w$, which is analytic in $U$ and for which $w(0)=0,|w(z)|<1$ for $z \in U$, such that $f(z)=g(w(z))$, for all $z \in U$. We denote by $\prec$ the subordination relation.

Definition 2.2. [3] Let $Q$ be the class of analytic functions $q$ in $U$ which has the property that are analytic and injective on $\bar{U} \backslash E(q)$, where

$$
E(q)=\left\{\zeta \in \partial U: \lim _{z \longrightarrow \zeta} q(z)=\infty\right\}
$$

and are such that $q^{\prime}(\zeta) \neq 0$ for $\zeta \in \partial U \backslash E(q)$.

Lemma 2.1. [2,3] Let $q \in Q$, with $q(0)=a$, and let $p(z)=a+a_{n} z^{n}+\ldots$ be analytic in $U$ with $p(z) \not \equiv a$ and $n \geq 1$. If $p \nprec q$, then there are two points $z_{0}=r_{0} e^{i \theta_{0}} \in U$, and $\zeta_{0} \in \partial U \backslash E(q)$ and a number $m \geq n \geq 1$ for which $p\left(U_{r_{0}}\right) \subset q(U)$,

(i) $\quad p\left(z_{0}\right)=q\left(\zeta_{0}\right)$

(ii) $z_{0} p^{\prime}\left(z_{0}\right)=m \zeta_{0} q^{\prime}\left(\zeta_{0}\right)$

(iii) $\operatorname{Re} \frac{z_{0} p^{\prime \prime}\left(z_{0}\right)}{p^{\prime}\left(z_{0}\right)}+1 \geq m \operatorname{Re}\left(\frac{\zeta_{0} q^{\prime \prime}\left(\zeta_{0}\right)}{q^{\prime}\left(\zeta_{0}\right)}+1\right)$.

The following result is a particular case of Lemma 2.1.

Lemma 2.2. $[2,3]$ Let $p(z)=1+a_{n} z^{n}+\ldots$ be analytic in $U$ with $p(z) \not \equiv 1$ and $n \geq 1$. If $\operatorname{Re} p(z) \ngtr 0, z \in U$, then there is a point $z_{0} \in U$, and there are two real numbers $x, y \in \mathbb{R}$ such that

(i) $p\left(z_{0}\right)=i x$

(ii) $z_{0} p^{\prime}\left(z_{0}\right)=y \leq-\frac{n\left(x^{2}+1\right)}{2}$,

(iii) $\operatorname{Re} z_{0}^{2} p^{\prime \prime}\left(z_{0}\right)+z_{0} p^{\prime}\left(z_{0}\right) \leq 0$. 
If $f(z)=z-\sum_{j=2}^{\infty} a_{j} z^{j}$, using the Noor and Sălăgean integral operators we define a new operator as follows:

$$
I_{N S}^{n} f(z)=I_{N}^{n} f(z) * I_{S}^{n} f(z)=z-\sum_{j=2}^{\infty} \frac{a_{j}^{2}}{j^{n} C(n, j)} z^{j},
$$

where $C(n, j)=\frac{(n+j-1) !}{n !(j-1) !}$ and $n \in \mathbb{N}_{0}$.

Remark 2.1. Differentiate the relation (2.1), we get

$$
\left[I_{N S}^{n} f(z)\right]^{\prime}=1-\sum_{j=2}^{\infty} \frac{a_{j}^{2}}{j^{n-1} C(n, j)} z^{j-1} .
$$

Multiplicating the equality (2.2) with $\frac{z}{n}$ we obtain

$$
\frac{z}{n}\left[I_{N S}^{n} f(z)\right]^{\prime}=\frac{z}{n}-\sum_{j=2}^{\infty} \frac{a_{j}^{2}}{n j^{n-1} C(n, j)} z^{j},
$$

which is equivalent to

$$
\frac{z}{n}\left[I_{N S}^{n} f(z)\right]^{\prime}+\frac{z}{n}(n-1)=z-\sum_{j=2}^{\infty} \frac{a_{j}^{2}}{n j^{n-1} C(n, j)} z^{j} .
$$

Now let $g \in T$ and $g(z)=z-\sum_{j=2}^{\infty}(n+j-1) z^{j}$. Then from (2.3), we obtain the following relation between $I_{N S}^{n-1} f(z)$ and $I_{N S}^{n} f(z)$ operators:

$$
I_{N S}^{n-1} f(z)=\frac{z}{n}\left[I_{N S}^{n} f(z)\right]^{\prime} * g(z)+\frac{n-1}{n} z * g(z)
$$

Using the Noor-Sălăgean integral operator, we define the following class of analytic functions:

Definition 2.3. A function $f \in T$ belongs to the class $C_{N S}(\alpha)$ if

$$
\operatorname{Re} \frac{z\left[I_{N S}^{n} f(z)\right]^{\prime}}{I_{N S}^{n} f(z)}>\alpha
$$

where $\alpha \in[0,1)$ and $z \in U$.

\section{Main Results}

Theorem 3.1. Let $f(z)=z-\sum_{j=2}^{\infty} a_{j} z^{j}$. Then $f \in C_{N S}(\alpha)$ if and only if

$$
\sum_{j=2}^{\infty} \frac{a_{j}^{2}}{j^{n-1} C(n, j)}\left[1-\frac{\alpha}{j}\right]<1-\alpha
$$


Proof. Let $f \in C_{N S}(\alpha)$, then we have

$$
\operatorname{Re} \frac{z\left[I_{N S}^{n} f(z)\right]^{\prime}}{I_{N S}^{n} f(z)}>\alpha, z \in U .
$$

If $z \in[0,1)$, we obtain

$$
\frac{z-\sum_{j=2}^{\infty} \frac{a_{j}^{2}}{j^{n-1} C(n, j)} z^{j}}{z-\sum_{j=2}^{\infty} \frac{a_{j}^{2}}{j^{n} C(n, j)} z^{j}}>\alpha .
$$

Since the denominator of (3.2) is positive, the relation (3.2) is equivalent with

$$
\alpha-1<\sum_{j=2}^{\infty}\left[\frac{\alpha a_{j}^{2}}{j^{n} C(n, j)} z^{j-1}-\frac{a_{j}^{2}}{j^{n-1} C(n, j)} z^{j-1}\right],
$$

and finally we get

$$
\alpha-1<\sum_{j=2}^{\infty} \frac{a_{j}^{2}}{j^{n-1} C(n, j)} z^{j-1}\left[\frac{\alpha}{j}-1\right] .
$$

Considering $z \rightarrow 1^{-}$along to the real axis, we get:

$$
\alpha-1<\sum_{j=2}^{\infty} \frac{a_{j}^{2}}{j^{n-1} C(n, j)}\left[\frac{\alpha}{j}-1\right] .
$$

To prove the reciproc implication we consider $f$ with the form (1.1) and for which the (3.1) inequality holds.

The condition $\operatorname{Re} \frac{z\left[I_{N S}^{n} f(z)\right]^{\prime}}{I_{N S}^{n} f(z)}>\alpha$ is equivalent to

We have

$$
\alpha-\operatorname{Re}\left(\frac{z\left[I_{N S}^{n} f(z)\right]^{\prime}}{I_{N S}^{n} f(z)}-1\right)<1 .
$$

$$
\begin{gathered}
\alpha-\operatorname{Re}\left(\frac{z\left[I_{N S}^{n} f(z)\right]^{\prime}}{I_{N S}^{n} f(z)}-1\right) \leq \alpha+\left|\frac{z\left[I_{N S}^{n} f(z)\right]^{\prime}}{I_{N S}^{n} f(z)}-1\right| \\
=\alpha+\left|\frac{\sum_{j=2}^{\infty} \frac{a_{j}^{2}}{j^{n} C(n, j)} z^{j}-\sum_{j=2}^{\infty} \frac{a_{j}^{2}}{j^{n-1} C(n, j)} z^{j}}{z-\sum_{j=2}^{\infty} \frac{a_{j}^{2}}{j^{n} C(n, j)} z^{j}}\right|=\alpha+\left|\frac{\sum_{j=2}^{\infty} \frac{a_{j}^{2}}{j^{n-1} C(n, j)} z^{j-1}\left[\frac{1}{j}-1\right]}{1-\sum_{j=2}^{\infty} \frac{a_{j}^{2}}{j^{n} C(n, j)} z^{j-1}}\right| \\
\leq \alpha+\frac{\sum_{j=2}^{\infty} \frac{a_{j}^{2}}{j^{n-1} C(n, j)}|z|^{j-1}\left|\frac{1}{j}-1\right|}{1-\sum_{j=2}^{\infty} \frac{a_{j}^{2}}{j^{n} C(n, j)}|z|^{j-1}}<\alpha+\frac{\sum_{j=2}^{\infty} \frac{a_{j}^{2}}{j^{n-1} C(n, j)}\left[1-\frac{1}{j}\right]}{1-\sum_{j=2}^{\infty} \frac{a_{j}^{2}}{j^{n} C(n, j)}}
\end{gathered}
$$




$$
=\frac{\alpha+\sum_{j=2}^{\infty} \frac{a_{j}^{2}}{j^{n-1} C(n, j)}\left[1-\frac{1}{j}-\frac{\alpha}{j}\right]}{1-\sum_{j=2}^{\infty} \frac{a_{j}^{2}}{j^{n} C(n, j)}} .
$$

To finish our proof, we need to show

$$
\frac{\alpha+\sum_{j=2}^{\infty} \frac{a_{j}^{2}}{j^{n-1} C(n, j)}\left[1-\frac{1}{j}-\frac{\alpha}{j}\right]}{1-\sum_{j=2}^{\infty} \frac{a_{j}^{2}}{j^{n} C(n, j)}}<1 .
$$

The (3.3) inequality is equivalent to

$$
\sum_{j=2}^{\infty} \frac{a_{j}^{2}}{j^{n-1} C(n, j)}\left[1-\frac{\alpha}{j}\right]<1-\alpha,
$$

which is the (3.1) condition.

Let $E_{N S}(\alpha)$ be a subclass of $C_{N S}(\alpha)$. The class is defined as follows:

$$
E_{N S}(\alpha)=\left\{f \in T:\left|\frac{z\left[I_{N S}^{n} f(z)\right]^{\prime}}{I_{N S}^{n} f(z)}-1\right|<1-2 \alpha \text { and } \alpha \in\left(0, \frac{1}{2}\right)\right\} .
$$

Theorem 3.2. Let $f \in T$ of the form (1.1). If $f \in E_{N S}(\alpha)$, then $\operatorname{Re} \frac{I_{N S}^{n} f(z)}{z}>0$. Proof. Suppose $f \in E_{N S}(\alpha)$. Then

$$
\left|\frac{z\left[I_{N S}^{n} f(z)\right]^{\prime}}{I_{N S}^{n} f(z)}-1\right|<1-2 \alpha .
$$

Let

$$
I_{N S}^{n} f(z)=z p(z)
$$

Differentiate (3.7), we obtain

$$
\left[I_{N S}^{n} f(z)\right]^{\prime}=z p^{\prime}(z)+p(z) .
$$

Then (3.6) is equivalent to

$$
\left|\frac{z p^{\prime}(z)}{p(z)}\right|<1-2 \alpha
$$

If the condition $\operatorname{Re} p(z)=\operatorname{Re} \frac{I_{N S}^{n} f(z)}{z}>0$ does not hold, then according to Lemma 2.2 , there is a point $z_{0} \in U$, and there are two real numbers $x, y \in \mathbb{R}$ such that

$$
p\left(z_{0}\right)=i x
$$


and

$$
z_{0} p^{\prime}\left(z_{0}\right)=y \leq-\frac{1+x^{2}}{2}
$$

These inequalities imply

$$
\left|\frac{z_{0} p^{\prime}\left(z_{0}\right)}{p\left(z_{0}\right)}\right|=\left|\frac{y}{i x}\right| \geq\left|\frac{\frac{1}{2}\left(1+x^{2}\right)}{x}\right|=\left|\frac{1}{2}\left(x+\frac{1}{x}\right)\right| \geq 1-2 \alpha .
$$

The above inequality contradicts (3.6) and consequently

$$
\operatorname{Re} p(z)=\operatorname{Re} \frac{I_{N S}^{n} f(z)}{z}>0
$$

where $z \in U$.

Theorem 3.3. Let

$$
F(z)=I_{c} f(z)=\frac{c+1}{z^{c}} \int_{0}^{z} f(t) t^{c-1} d t, c \in \mathbb{N} .
$$

If $f \in C_{N S}(\alpha)$, then $F=I_{c}(f) \in C_{N S}(\beta)$, where

$$
\beta=\beta(\alpha, 2)=1-\frac{(1-\alpha)(c+1)^{2}}{(c+2)^{2}(2-\alpha)-(c+1)^{2}(1-\alpha)}
$$

and $\beta>\alpha, \alpha \in[0,1)$.

Proof. Suppose $f \in C_{N S}(\alpha)$. Then by Theorem 3.1 we have

$$
\sum_{j=2}^{\infty} \frac{a_{j}^{2}(j-\alpha)}{j^{n} C(n, j)(1-\alpha)}<1
$$

We know that if $f$ has the form (1.1), then

$$
F(z)=\frac{c+1}{z^{c}} \int_{0}^{z} f(t) t^{c-1} d t=z-\sum_{j=2}^{\infty} \frac{c+1}{c+j} a_{j} z^{j},
$$

and to prove that $F \in C_{N S}(\beta)$ is sufficient to have

$$
\sum_{j=2}^{\infty} \frac{j-\beta}{j^{n} C(n, j)(1-\beta)}\left(\frac{c+1}{c+j}\right)^{2} a_{j}^{2}<1 .
$$

This last inequality is implied by

$$
\frac{j-\beta}{1-\beta} \cdot \frac{(c+1)^{2} a_{j}^{2}}{j^{n} C(n, j)(c+j)^{2}} \leq \frac{j-\alpha}{1-\alpha} \cdot \frac{a_{j}^{2}}{j^{n} C(n, j)},
$$


for all $j \in \mathbb{N}$ and $j \geq 2$.

From (3.10) we deduce that

$$
\beta \leq 1-\frac{(1-\alpha)(c+1)^{2}(j-1)}{(c+j)^{2}(j-\alpha)-(c+1)^{2}(1-\alpha)}=\beta(\alpha, j),
$$

$j \in \mathbb{N}, j \geq 2$. We will prove that

$$
\beta(\alpha, j) \geq \beta(\alpha, 2), j \in \mathbb{N}, j \geq 2
$$

Let consider the function $\varphi:[2, \infty) \rightarrow \mathbb{R}$

$$
\varphi(x)=\frac{x-1}{(x+c)^{2}(x-\alpha)-(c+1)^{2}(1-\alpha)}, x \in[2, \infty) .
$$

Then

$$
\varphi^{\prime}(x)=\frac{g(x)}{\left[(x+c)^{2}(x-\alpha)-(c+1)^{2}(1-\alpha)\right]^{2}},
$$

where $g(x)=-2 x^{3}+(3-2 c-\alpha) x^{2}+(4 c-2 \alpha) x-2 c-(1-\alpha)$.

We have

$$
\begin{gathered}
g^{\prime}(x)=-6 x^{2}+2(3-2 c-\alpha) x+4 c-2 \alpha, \\
g^{\prime \prime}(x)=-12 x+6-4 c-2 \alpha<0,
\end{gathered}
$$

$x \in[2, \infty)$. Then

$$
g^{\prime}(x) \leq g^{\prime}(2)=-12-4 c-6 \alpha<0, x \in[2, \infty)
$$

and

$$
g(x) \leq g(2)=-4-8 \alpha-2 c-(1-\alpha)<0, x \in[2, \infty) .
$$

We obtain $\varphi^{\prime}(x)<0, x \in[2, \infty)$ and from this

$$
\beta(\alpha, j)=1-\varphi(j)(1-\alpha)(c+1)^{2} \geq 1-\varphi(2)(1-\alpha)(c+1)^{2}=\beta(\alpha, 2,)
$$

where $\beta(\alpha, 2)$ is given by (3.9). Finally $\beta>\alpha$ is equivalent to

$$
1-\alpha>\frac{(1-\alpha)(c+1)^{2}}{(c+2)^{2}(2-\alpha)-(c+1)^{2}(1-\alpha)} .
$$

\section{Acknowledgements}

The authors are grateful to Professor Róbert Szász and Professor Grigore Şt. Sălăgean for their valuable comments which improved the presentation of the paper. The authors also thank the referees for their useful comments. 


\section{References}

[1] M. Darus, R.W. Ibrahim, Partial sums of analytic functions of bounded turning with applications, Comput. Appl. Math. 29 (1) (2010) 81-88.

[2] S.S. Miller, P.T. Mocanu, Differential Subordinations Theory and Applications, Marcel Dekker, New York, Basel, 2000.

[3] P.T. Mocanu, T. Bulboacă, G.Şt. Sălăgean, Teoria Geometrică a Funcţiilor Univalente, Ed. a II-a, Casa Cărţii de Ştiinţă, Cluj-Napoca, 2006, 460+9 pag., ISBN 973-686-959-8 (romanian only).

[4] K.I. Noor, M.A. Noor, On integral operators, J. Math. Anal. Appl. 238 (1999) 341-352.

[5] K.I. Noor, On new classes of integral operators, J. Natur. Geom. 16 (1999) 71-80.

[6] H. Silverman, A survey with open problems on univalent functions whose coeffcients are negative, Rocky Montain J. Math. 21 (1991) 1099-1125.

[7] G.Şt. Sălăgean, Subclasses of univalent functions, Lecturer Notes in Math. (Springer Verlag) 1013 (1983) 362-372.

DOI: $10.7862 / \mathrm{rf} .2016 .4$

Olga Engel - corresponding author

email: engel_olga@hotmail.com

Department of Mathematics,

Babeş - Bolyai University,

Cluj - Napoca,

Romania.

Yao Liang Chung,

email: chungyaoliang@gmail.com

School of Mathematical Sciences,

Universiti Sains Malaysia,

11800 Penang,

Malaysia.

Received 12.02.2016

Accepted 10.04.2016 